\title{
Fetal Pulsed-Wave Doppler Atrioventricular Activity Detection by Envelope Extraction and Processing
}

\author{
Eleonora Sulas ${ }^{1}$, Emanuele Ortu ${ }^{1}$, Monica Urru ${ }^{2}$, Alessandra Cadoni ${ }^{2}$, Roberto Tumbarello ${ }^{2}$, Luigi \\ Raffo $^{1}$, Danilo Pani ${ }^{1}$ \\ ${ }^{1}$ Department of Electrical and Electronic Engineering, University of Cagliari, Italy \\ ${ }^{2}$ Division of Paediatric Cardiology, S. Michele Hospital, Cagliari, Italy
}

\begin{abstract}
Pulsed-Wave Doppler (PWD) is a diagnostic ultrasound technique widely used for fetal heart rate monitoring. Fetal PWD is particularly challenging since, beyond being intrinsically operator-dependent, different issues related to the fetal heart size, the fetal movements and the ultrasound artifacts appear. In long PWD recordings, the signal segments completely meaningful for a morphological analysis, i.e. including a readable atrial and ventricular activity, are then limited in number and duration.

In this work, an approach for the automatic detection of the meaningful fetal cardiac activity from $P W D$ video recordings is presented and evaluated, using the annotations made by an expert cardiologist. It consists of the video pre-processing for image thresholding, filtering and envelope extraction by edge detection, and a supervised classification stage. A dataset including 30 signals from 17 pregnant women was adopted, extracting from it multiple segments, including different quality recordings. A supervised classification approach for the detection of the signal segments completely meaningful for a morphological analysis was then applied, revealing an accuracy greater than $99 \%$.
\end{abstract}

\section{Introduction}

The guidelines for the management of fetal heart cardiac defects (CDH), by the American Heart Association (AHA), pinpoint how the diagnosis of cardiac disease in the fetus should be mostly performed by means of echocardiography, which represents the first-line tool for diagnosis [1]. Diagnostic ultrasound systems that could be exploited for this purpose include B-mode, M-mode, colour and pulsed-wave Doppler (PWD). In particular, through the PWD examination, it is possible to evaluate the blood flow velocity through the heart valves.

PWD allows estimating the velocity of the moving blood in a defined sampled volume. Using an apical window, commonly used in everyday clinical practice, the beam is positioned as parallel as possible to the direction of the blood flow that goes into the mitral valve. Using a 5-chamber apical view, the four chambers and the aortic region can be seen. PWD is an operator dependent method that is usually characterized by several noises, such as valve clicks, mirror image effects, vascular motion artefacts and wall artefacts, making difficult the detection of the important patterns of this technique. A previous method [2] proposed the automatic recognition of complete atrioventricular activity in fetal pulsed wave Doppler signal using an artificial neural network (ANN). This work represents the evolution of the aforementioned method, leading to very high accuracy. For the technique validation, 30 traces from 17 different pregnant women are used. All the traces were labelled from an expert cardiologist.

\section{Materials and Methods}

The present study was approved by the Independent Ethical Committee of the Cagliari University Hospital (AOU Cagliari), and performed following the principles outlined in the Helsinki Declaration of 1975, as revised in 2000. All of the volunteers provided their signed informed consent to the protocol.

\subsection{Pulsed-wave Doppler Ultrasound}

During the everyday clinical practice, for routine Doppler examination, the apical window is used. On healthy subjects, this window allows obtaining a PWD with a well-known pattern, shown in Fig. 1. In presence of cardiac heart diseases, the flow patterns are different. The normal pattern consists of three main epochs: the $\mathrm{E}$ wave, which represents the blood flow through the mitral valve 
during passive ventricular filling, the A wave, which represents the blood flow velocity during active ventricular filling due to the atrial contraction, and the $\mathrm{V}$ wave, following them in opposite polarity. The $\mathrm{V}$ wave identifies the blood flow through the aortic valve due to ventricular activity.

In this work, PWD was acquired by a Philips iE33 ultrasound device. In order to digitalize the PWD video at a frame rate of $60 \mathrm{~Hz}$, a StarTech USB3HDCAP USB3.0 Video Capture Device was connected to the DVI output of the iE33.

\subsection{The Proposed Method}

The data processing was performed using Matlab v2017b. In order to analyse the signal for the identification of the main E, A and V waves, the following main steps were applied:

1. Envelope extraction: the whole video was converted into a single wide image (Fig. 2) and the PWD envelope was extracted in the form of two 1D signals (upper and lower parts),

2. Pattern recognition: an artificial neural network (ANN) was trained to automatically recognize the complete and readable full beats in the signal.

The different steps are separately analysed hereafter.

\subsubsection{Envelope Extraction}

The first step consists in identifying the boundary that separates the PWD spectrum from the background. The PWD spectrum covers a large part of the grayscale range. To effectively separate the information of interest, the image was binarized using a global threshold obtained from a gray-level-median histogram based on twodimensional (2D) Otsu's method [3]. This threshold was selected as the most efficient and robust, in terms of noise and information contained in an image, after a comparison against the original Otsu's method and a fixed manual threshold [2]. The former, works well in bimodal histogram image, but not in this kind of image. For this reason, in this case, it resulted in a high threshold, deleting significant information from the images. The latter reached better results than the former, by detecting also the information of the darker pixels. However, 2D Otsu's method allows achieving the better results on the largest part of the tested images.

The binarized image was than processed with a morphological operation, known as area opening, to remove all 4-connected components having a small area (70 pixels), producing another binary image. The parameters for this processing produced the best results compared to different area opening operations. This step is very important to reduce noise and to remove spurious elements that could hamper the envelope extraction.

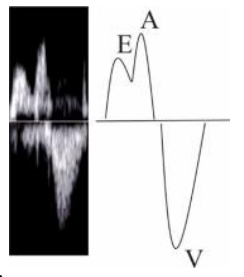

Figure 1: PWD waveform

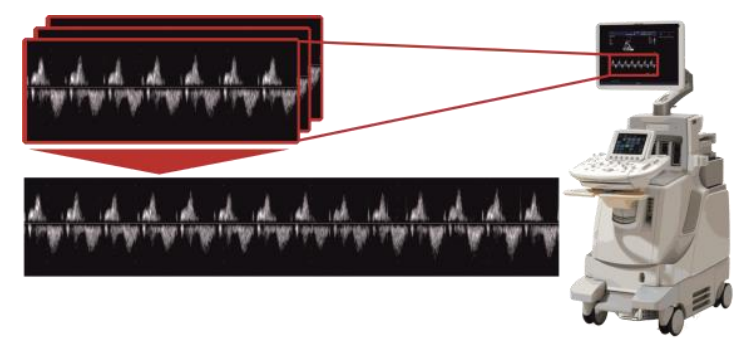

Figure 2: Construction of a single wide image from different PWD signals.

However, residual ultrasound artifacts may still hide the actual information of the cardiac cycle, so that fiducial points are not easily detectable on the image. For this reason, rather than working on the extraction of features from the image, the idea behind also the first proposed approach [2] was to reduce the complexity of the feature extraction problem by converting the envelope of the binarized PWD velocity spectrum into two different curves representing respectively its upper $G_{u}(x)$ and lower $G_{l}(x)$ profiles. Such curves are obtained as in [2] by considering the first white pixel for $G_{u}(x)$ and the last one for $G_{l}(x)$, for each column of the image.

Fig. 3 shows the PW Doppler signals with the associated $G_{u}$ and $G_{l}$ curves.

\subsubsection{Pattern Recognition}

Due to the variable position of the fetus within the maternal uterus, the mitral blood inflow can be direct towards the ultrasound transducer or moving away from it, causing a positive or negative EA wave. While in the previous work [2] any segment of the estimated Doppler envelope could be classified in three different ways depending on the EA-V existence and balance, in this work, the user has to give an input and to tell if the EA wave is negative. In this case, the image will be flip. Consequently, only two classes are considered: to be a complete fetal beat, called EAV, or an incomplete/ unrecognized beat, hereafter called ND.

In this work, an ANN characterized by 264 input nodes, 10 hidden nodes and 2 output nodes was trained using the scaled conjugate gradient backpropagation algorithm. The number of neurons in the hidden layer was empirically chosen in order to obtain acceptable performance on the available dataset without incurring in the overfitting 
problem.

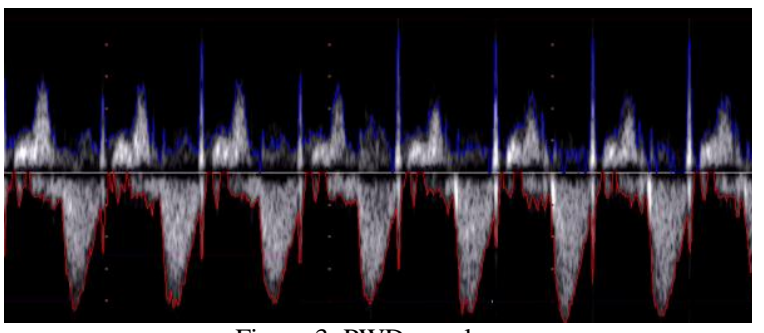

Figure 3: PWD envelope.

The number of inputs of the ANN depends on the size of the feature vector. In this work, the ANN is feed by windows of the two vectors $G_{u}(x)$ and $G_{l}(x)$, without any resampling, in order to avoid losing any information, but still normalizing them respectively between 0 and 1 and between -1 and 0 [2]. The windows length was chosen in order to be the most representative length of a cardiac cycle of the fetus between the $21^{\text {st }}$ to the $27^{\text {th }}$ gestational week. A healthy fetus of that gestation age could have a heart rate ranging from 110 to $160 \mathrm{bpm}$ [4], so considering a sampling frequency for the envelope signals resulting from the video equal to $284 \mathrm{~Hz}$, a length of 128 samples was chosen. Other four area features were used, the area under the curve of the first 64 samples of $G_{u}(x)$ and $G_{l}(x)$, and the area under the remaining 64 samples of both signal. Further four features, based on the pixels analysis, were added in order to improve the classifier performance. The brightness information is very useful for the recognition of the meaningful part of the image. In fact, ultrasound artifacts could be less bright than the interesting parts of PWD and, moreover, the cardiac cycle parts are characterized by the same brightness in the same trace. For this reason, the mean values of the pixel included in the aforementioned four areas, were computed. Overall, 264 input features are chosen, 128 samples of $G_{u}$ and $G_{l}, 4$ area features and 4 pixel features, in order to characterize as better as possible the patterns.

In order to train and validate the ANN, a dataset involving 17 low-risk voluntary pregnant women $\left(21^{\text {st }}\right.$ to $27^{\text {th }}$ gestational weeks) was acquired. For each subject, a different number of traces were analyzed. Such traces can be considered independent each other because they were recorded by changing the position of the ultrasound probe. Details about the study population are given in Table I.

Classification was performed by means of the Matlab Neural Network Toolbox. The validation of the ANN was performed choosing a 30-fold scheme, carrying out the validation on a different trace per fold, not used for training. An expert cardiologist, using a custom interface, annotated the windows representing a complete fetal cardiac cycle. Overall, 1950 windows from 30 PWD videos taken from the 17 voluntary pregnant volunteers were extracted and annotated from the cardiologist. Other 1950 windows representing incomplete or malformed fetal
TABle 1. Study Population

\begin{tabular}{|c|c|c|}
\hline Subject & $\begin{array}{c}\text { Gestational } \\
\text { age }\end{array}$ & \#traces \\
\hline 1 & 27 weeks & 3 \\
\hline 2 & 23 weeks+5 & 1 \\
\hline 3 & 21 weeks+6 & 1 \\
\hline 4 & 23 weeks+4 & 2 \\
\hline 5 & 24 weeks+2 & 1 \\
\hline 6 & 24 weeks & 2 \\
\hline 7 & 23 weeks+4 & 3 \\
\hline 8 & 25 weeks+1 & 1 \\
\hline 9 & 24 weeks & 1 \\
\hline 10 & 27 weeks & 2 \\
\hline 11 & 25 weeks & 2 \\
\hline 12 & 21 weeks+1 & 2 \\
\hline 13 & 22 weeks +4 & 1 \\
\hline 14 & 24 weeks +4 & 3 \\
\hline 15 & 25 weeks+4 & 2 \\
\hline 16 & $21+5$ & 2 \\
\hline 17 & 25 weeks+2 & 1 \\
\hline
\end{tabular}

cardiac cycles were taken randomly to be included in the training set. Overall, 3900 windows were used for training, validation and test of the developed ANN. Such an approach led to a balanced dataset.

To assess the performance of the classifier, the true positive rate (TPR), or Sensitivity, the true negative rate (TNR), or Specificity, and accuracy (Acc) were computed.

\section{Results}

Fig. 4 shows the box-plot of the result of the 30-fold scheme validation. The median value is $100 \%$ for all the quality performance metrics, whereas only specificity is

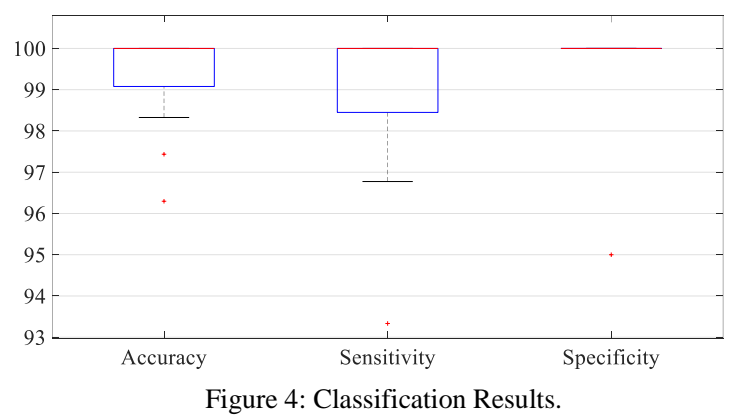

$100 \%$ for all the folds but a single outlier.

Overall, the accuracy resulted to be very high, reaching in most of the case $100 \%(99.5 \pm 0.9)$, confirming the better quality of the proposed approach compared to the first method presented in [2], that reached an accuracy up to $88 \%$, and on average, a sensitivity and a specificity 
respectively of $89 \%(99.2 \pm 1.5$ in this work) and $88.5 \%$ $(99.8 \pm 0.9$ in this work). Now, the method ability to identify the beats describing a complete atrioventricular cycle, not confounding them with incomplete or malformed ones, is very high, regardless the low quality of some traces, that in the previous work brought to the worse results.

\section{Conclusions}

In this work, a method for the automatic detection of complete atrioventricular activity in PWD signals was presented and evaluated on real signals from 17 pregnant women. The method, exploiting a supervised classifier trained on features extracted from the PWD envelope and brightness information, achieves on average remarkably high performance.

Such a result opens to the possibility of using the proposed approach as a first step for the evaluation of important parameters which can be automatically computed on the PWD traces to help the diagnosis and the monitoring of the CHD. First, the fetus heart rate can be detected, leading to the monitoring of fetus bradycardia or tachycardia [5]. The Atrioventricular Conduction time can be computed, in order to evaluate a possible atrioventricular block [6]. An important index, such as the Fetal Modified Myocardial Performance Index, is a ratio of isovolumetric to ejection time cardiac time intervals [7], and can be obtained automatically. Currently, these parameters are being evaluated.

\section{Acknowledgements}

The authors wish to thank the team headed by Dr. Roberto Tumbarello for the important support. Eleonora Sulas is grateful to Sardinia Regional Government for supporting her PhD scholarship (P.O.R. F.S.E., European Social Fund 2014-2020).

\section{References}

[1] Donofrio MT, Moon-Grady AJ, Hornberger LK, et al. "Diagnosis and treatment of fetal cardiac disease: a scientific statement from the American Heart Association". Circulation. 2014; 129:2183-242.

[2] Sulas E., Ortu E., Raffo L., Urru M., Tumbarello R., Pani D. "Automatic recognition of complete atrioventricular activity in fetal pulsed wave doppler signals". EMBC 2018.

[3] Chunshi Sha, Jian Hou, Hongxia Cui, Jianxin Kang. "Gray level-median histogram based 2D Otsu's method". 2015 International Conference on Industrial InformaticsComputing Technology, Intelligent Technology, Industrial Information Integration.

[4] Pildner von Steinburg S., Boulesteix A., Lederer C., Grunow S., Schiermeier S., Hatzmann W., Schneider K.M., Daumer M. "What is the "normal" fetal heart rate?" PeerJ 2013.

[5] Weber R. et al. "Diagnosis and management of common fetal arrhythmias" Journal of the Saudi Heart Association 23, 6166. 2011.

[6] Tomek V. et al. "Atrioventricular conduction time in fetuses assessed by doppler echocardiography" Physiol. Res. 60: 611-616, 2011.

[7] Maheshwari P. et al. "The fetal modified myocardial performance index: is automation the future?" Hindawi Publishing Corporation BioMed Research International Volume 2015, Article ID 215910, 9 pages.

Address for correspondence.

Name: Eleonora Sulas

Full postal address: P.zza d'Armi, 09123 Cagliari, Italy

E-mail address (optional): eleonora.sulas@diee.unica.it 